\title{
A Case of Pure Mucinous Breast Carcinoma in a 25-Year-Old Female Who Showed Complete Pathological Response to Neoadjuvant Chemotherapy despite Poor Clinical Response
}

\author{
Pei Du ${ }^{a}$ Chunjie $\mathrm{Hou}^{\mathrm{a}}$ Jinglan Tang ${ }^{\mathrm{a}}$ Ying Liu ${ }^{\mathrm{a}}$ Qiaohong $\mathrm{Hu}^{\mathrm{a}}$ \\ Hongfeng $\mathrm{He}^{\mathrm{a}}$ Kefeng $\mathrm{Lu}^{\mathrm{a}}$ Lucou Chen $^{\mathrm{b}}$ \\ a Department of Ultrasound, Zhejiang Provincial People's Hospital, People's Hospital of Hangzhou Medical College, \\ Hangzhou, China; bepartment of Ultrasound, Tiantai People's Hospital of Zhejiang Province, Tiantai Branch of \\ Zhejiang Provincial People's Hospital, Taizhou, China
}

\section{Established Facts}

- Mucinous breast carcinoma is a rare histological subtype in young patients and usually presents without lymph node involvement.

- The pathological characterization of response to neoadjuvant chemotherapy (NAC) in mucinous breast carcinoma is rarely reported.

\section{Novel Insights}

- This case report presents pure mucinous breast carcinoma in a 25-year-old female with axillary lymph node metastasis.

- Complete pathological response of pure mucinous breast carcinoma to NAC was found despite poor clinical response.

\section{Keywords}

Mucinous breast carcinoma - Neoadjuvant chemotherapy . Complete pathological response

\footnotetext{
Abstract

Introduction: Mucinous breast carcinoma is a rare histologic subtype of primary breast cancers accounting for 1-6\%. It is a rare histological variant in young patients and usually presents without lymph node involvement, and its pathological response to neoadjuvant chemotherapy is rarely reported. Case Presentation: Pure mucinous breast carcinoma in a 25-year-old female was treated with neoadjuvant chemotherapy every 3 weeks for 8 cycles. After the fifth cy-
}

cle, the mass size showed no change. We performed modified radical mastectomy in the left breast and axillary lymph node clearance. However, the pathological report showed a complete elimination of both the breast tumor and axillary lymph nodes, which were filled with mucus but did not contain malignant cells Discussion: Chemotherapy was profoundly effective against the tumor cells, but ineffective against large amounts of extracellular mucus. Even though the cancer cells were sensitive to chemotherapy, the volume of mucinous cancer couldnot be reduced. Conclusion: In summary, the evaluation criteria of tumor response to chemotherapy based on maximum diameter only should be considered insufficient for mucinous carcinoma.

(C) 2019 S. Karger AG, Base 


\section{Introduction}

Mucinous carcinoma of the breast (MBC) is a rare histologic subtype accounting for 1-6\% of all primary breast cancers [1]. These tumors frequently develop in postmenopausal females and are rarely reported in young patients $[1,2]$. They usually have a better prognosis without lymph node involvement in contrast to the non-special type $[3,4]$. Neoadjuvant chemotherapy (NAC) is the standard of care for patients with stages II and III breast cancer who might benefit from breast-conserving surgery after downstaging of their disease [5-7]. Response and histological changes after NAC have been widely reported, mostly in invasive ductal carcinoma. However, NAC is not usually used in mucinous carcinomas because they present as small, slow-growing, and human epidermal growth factor receptor 2 (HER2) negative $[4,5]$ tumors. Therefore, there is a lack of pathological characterization of response to NAC in MBC. In this study, we report a 25-year-old Chinese female with a pure mucinous breast carcinoma (PMBC) and axillary lymph node metastasis who showed pathological complete response (pCR) to NAC despite poor clinical response.

\section{Case Presentation}

A 25-year-old female presented with a 3-year history of a slow-growing mass in her left breast. Her past medical history was unremarkable. Clinical examination revealed a large regular mass in the upper outer quadrant of the left breast and palpable ipsilateral axillary active lymph nodes. Ultrasonography revealed an irregularly shaped, hypoechoic lesion with mixed solid and cystic components at the 12-10'clock position in the left breast, measuring $60 \times 18 \times 45 \mathrm{~mm}$ in diameter (Fig. 1). In addition, there were three abnormal left axillary lymph nodes, suggestive of significant lymph node metastasis. Magnetic resonance imaging (MRI) revealed a hypointense, irregular mass measuring $58 \times 20 \mathrm{~mm}$ in the left breast laterally that showed mild enhancement in contrast sequences and a slightly hyperintense sequence on T2 and multiple enhanced left axillary lymph nodes (Fig. 2). The patient underwent core needle biopsy of the left breast mass and fine-needle biopsy of the nodes in our hospital (Fig. 3), and both showed that at least $90 \%$ of the contents in PMBC were mucinous. Immunohistochemical analysis of the left axillary lymph node resection biopsy revealed that $90 \%$ of the tumor cells were strongly positive for estrogen receptor (ER) and progesterone receptor (PR), but negative for HER2 (Fig. 3). The patient's TNM staging was CT3N1M0, and stage IIIA was confirmed [8]. Therefore, she received NAC consisting of 4 cycles of epirubicin $90 \mathrm{mg} /$ $\mathrm{m}^{2}$ (intravenous) plus cyclophosphamide $600 \mathrm{mg} / \mathrm{m}^{2}$ (intravenous), followed by 4 cycles of paclitaxel $180 \mathrm{mg} / \mathrm{m}^{2}$ (intravenous) every 3 weeks prior to surgery. After cycle 4 ( 12 weeks), the tumor size remained unchanged by breast ultrasound examination. MRI revealed an unenhanced mass in the left breast, approximately $60 \mathrm{~mm}$ in diameter, and a higher hyperintense sequence on T2 (Fig. 2). After cycle 5 (15 weeks), ultrasound examination showed that the mass size was still unchanged. Based on the patient's strong wish, we performed modified radical mastectomy (retains the areola and nipple) of the left breast and axillary nodes

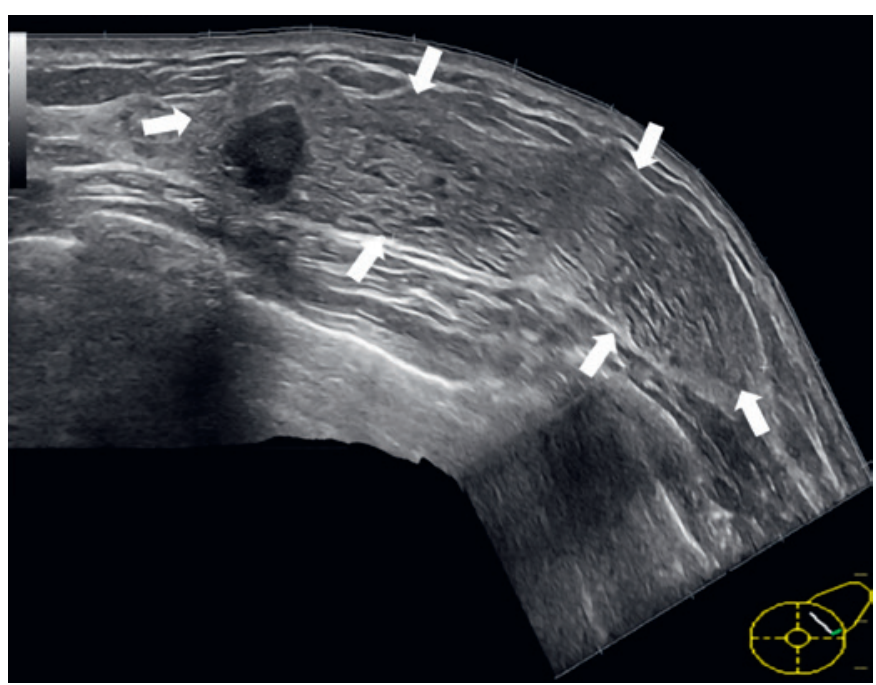

Fig. 1. a Ultrasonography revealing an irregularly shaped, hypoechoic lesion with mixed solid and cystic components at the $12-10^{\prime}$ clock position in the left breast, measuring $>5 \mathrm{~cm}$ in diameter (white arrows).

clearance in November 2018. The pathological report showed a complete elimination of both the breast tumor and axillary lymph nodes, which were filled with mucus but did not contain malignant cells (Fig. 4). A $60-\mathrm{mm}$ mucin lake matched the original tumor size. Mucin was also seen in 2 of the 6 axillary lymph nodes, both of which were filled with mucus and were thought to be a response to chemotherapy. Interestingly, residual mucin was co-located with the initial tumor in the breast as well as in the axillary lymph nodes. The pathological assessment of the therapeutic response was pCR $[9,10]$, pathological staging was ypTON0M0 $[5,11]$. Postoperatively, she continued to receive the remaining 3 cycles of paclitaxel $\left(180 \mathrm{mg} / \mathrm{m}^{2}\right)$ every 3 weeks. The chemotherapeutic course was completed in February 2019, and the toxicities were tolerable. She then received adjuvant radiotherapy. The patient is alive for 15 months from the initial diagnosis, without evidence of relapse.

\section{Discussion}

This case report is interesting for two reasons: first, it shows a PMBC in a 25 -year-old female with axillary lymph node metastasis, which is a rare variant reported in young patients; second, although the clinical response was poor, $\mathrm{pCR}$ to NAC was found in this $\mathrm{PMBC}$ case.

$\mathrm{MBC}$ is a rare entity, representing $1-6 \%$ of the invasive breast carcinomas. It is defined as nests of cells floating in lakes of mucin [1]. There are two histologic subtypes: pure mucinous carcinoma when mucous components account for $>90 \%$ of the tumors, and mixed mucinous carcinoma when the mucin is $>50$ but $<90 \%$ $[11,12]$. In this case, the core biopsy showed that $>90 \%$ of the tumor tissue was mucus, consistent with the diagnosis of PMBC. Previous studies reported that PMBC is frequently found in postmenopausal patients, and its 
Fig. 2. a, b Contrast-enhanced MRI scan showing mild enhancement in both the left breast tumor and the left axillary lymph nodes (prior to chemotherapy). c, d Contrast-enhanced MRI scan showing no enhancement in both the left breast tumor and the left axillary lymph nodes (after chemotherapy).

Fig. 3. Core biopsy. a Panoramic view of the tumor that is composed of multiple groups of neoplastic cells immersed in extracellular mucin $(\times 4)$. b A left axillary swollen lymph node resection biopsy showing mucin lakes with cellular characteristics of the tumor $(\times 20)$. c Ninety percent of the neoplastic cells are ER positive $(\times 40)$. d Ninety percent of the neoplastic cells are PR positive $(\times 20)$. e Ninety percent of the neoplastic cells are HER-2 negative $(\times 10)$.
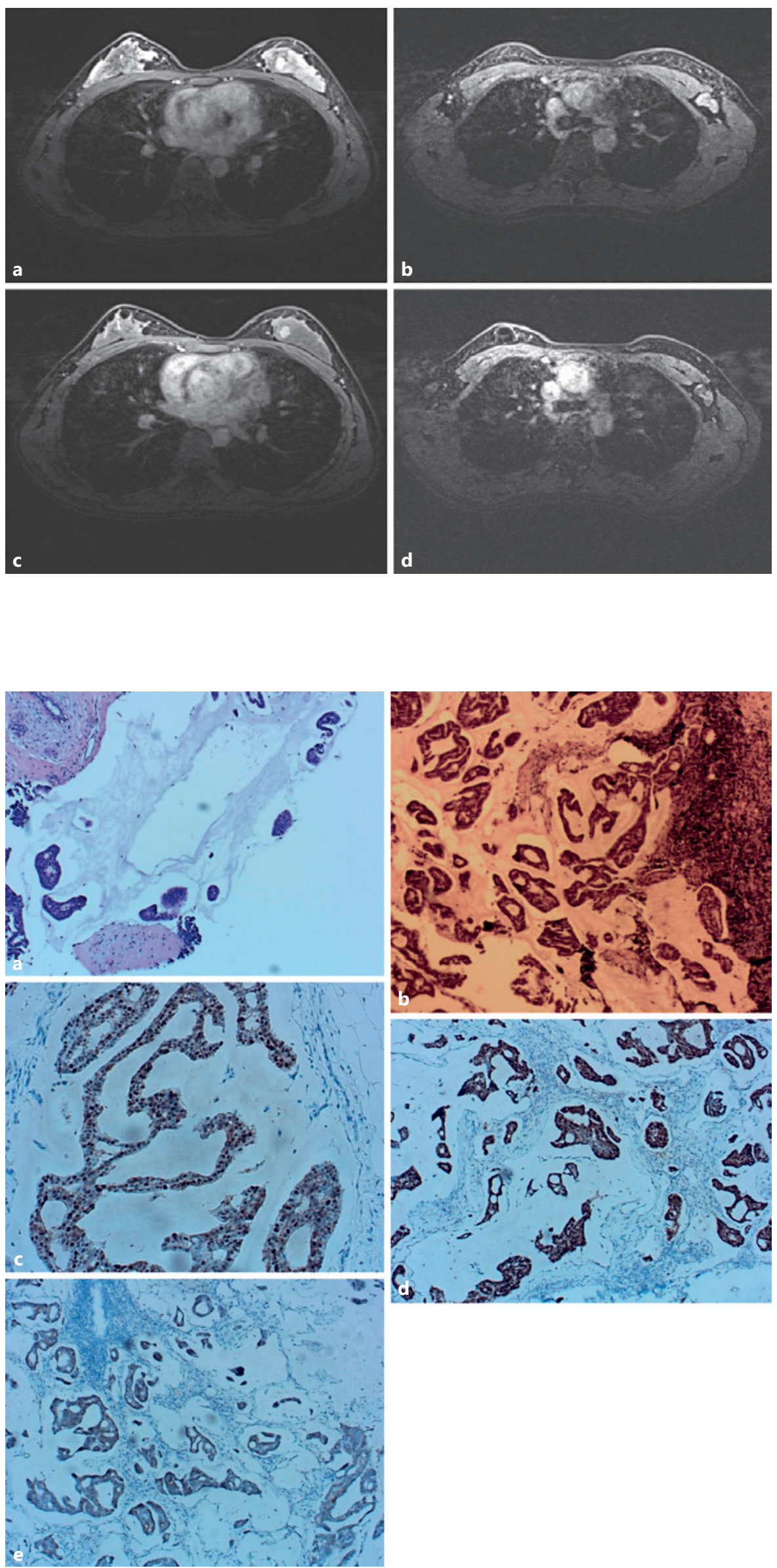

an sin 


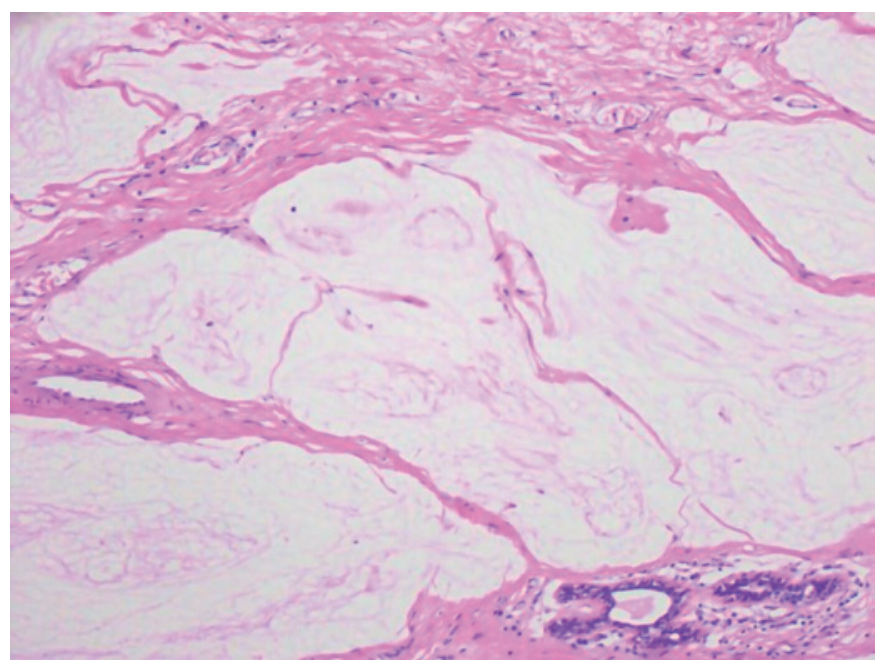

Fig. 4. Surgical resection. Mucinous lakes with no tumor cells in the breast tumor after NAC treatment $(\times 4)$, corresponding to $\mathrm{pCR}$.

prevalence in females younger than 35 years is $1 \%[2,13]$. MBC could be easily misdiagnosed as benign lesions because of its slow growth and well-circumscribed margins $[2,14]$, which are classified as low-grade tumors. Lymph node metastasis is rare, even with large primary pure or mixed MBC [11]. It was reported to be $<5 \%$ in $\mathrm{PMBC}$ patients with axillary lymph node metastasis, which implied a favorable prognosis [15]. However, the only poor prognostic factor is lymph node involvement $[1,16]$. Komenaka et al. [2] reported that the number of axillary lymph nodes involved was the only significant predictor of death. MBC shows a high probability of benignity but can also be a rare histological variation of a malignant tumor, which is not common in young patients. Therefore, a differential diagnosis of breast lumps in young patients is very important $[17,18]$.

NAC has become standard therapy for locally advanced or large operable breast cancer, leading to significant regression of cancer and thus making breastconserving surgery possible [5-7]. As we know, few literature reports present the typical responses of $\mathrm{MBC}$ to NAC. The typical immunoprofile for PMBC is ER and/ or PR positive and HER-2 negative [3, 4, 19]. These biological features suggest that NAC is not usually used in mucinous carcinoma $[4,5]$. However, our patient did not meet the above criteria, as she had a large breast mass with axillary lymph node metastasis that suggested a more aggressive cancer. Fortunately, she showed a pCR after NAC. Clinical and radiological evaluations of the response to NAC are based on residual tumor size, and the correlation with pathologic response is often inaccurate. Tumor size remains the gold standard for evaluating residual tumor after chemotherapy [20]. Rajan et al. [21] reported that chemotherapy can significantly re duce the number of cells but has little effect on the overall size of some tumors. In other words, chemotherapy has a strong inhibitory effect on tumor cells but is ineffective against large amounts of extracellular mucus. Therefore, mucus is an important component of the residual tumor. In addition, the residual tumor may be associated with chemotherapy-induced tumor cell necrosis, fibrosis, or fibrocystic changes. These secondary changes may lead to an overestimation of the residual tumor size at the macroscopic level [22]. This phenomenon results in a discrepancy between the residual tumor size and the effect of chemotherapy. However, the pathological diagnosis is usually made by core biopsy before chemotherapy that, however, represents a tiny proportion of the whole tumor. Therefore, it is difficult to determine whether these obvious changes reflect the heterogeneity of the primary tumor or the response to chemotherapy. Furthermore, based on our patient alone, we cannot recognize the general trend of chemotherapy response. However, pathological examination indicates that the size of mucinous carcinomas may not be reduced even if the tumor cells are sensitive to chemotherapy.

\section{Conclusion}

In summary, maximum diameter may be an in adequate criterium to evaluate mucinous carcinoma response to chemotherapy. More detailed studies are needed to evaluate the pathological response of mucinous breast carcinoma to NAC.

\section{Acknowledgement}

We would like to express our gratitude to all who helped writing the manuscript. Special thanks should go to Professor Chen, from whose guidance we benefited greatly. We are particularly indebted to Mr. Hou and Ms. Liu, who kindly encouraged us and provided useful instructions throughout the writing process. Finally, we wish to extend our thanks to the library assistants who supplied us with reference materials of great value.

\section{Statement of Ethics}

We declare that the patient agreed to the description and publication of her case (including the publication of the pictures).

\section{Disclosure Statement}

There are no conflicts of interest for all authors. 


\section{Funding Sources}

This work was supported by Public Welfare Projects of Science and Technology Department in Zhejiang Province, grant No. 2017C33097; Projects of Zhejiang Education Department, grant No. 201840393; and Projects of Medical and Health Technology Development Program in Zhejiang Province, grant No. 2019RC125.

\section{References}

1 Bae SY, Choi MY, Cho DH, Lee JE, Nam SJ, Yang JH. Mucinous carcinoma of the breast in comparison with invasive ductal carcinoma: clinicopathologic characteristics and prognosis. J Breast Cancer. 2011 Dec;14(4): 308-13.

2 Komenaka IK, El-Tamer MB, Troxel A, Hamele-Bena D, Joseph KA, Horowitz E, et al. Pure mucinous carcinoma of the breast. Am J Surg. 2004 Apr;187(4):528-32.

3 Yerushalmi R, Hayes MM, Gelmon KA. Breast carcinoma-rare types: review of the literature. Ann Oncol. 2009 Nov;20(11): 1763-70.

4 Barkley CR, Ligibel JA, Wong JS, Lipsitz S, Smith BL, Golshan M. Mucinous breast carcinoma: a large contemporary series. Am J Surg. 2008 Oct;196(4):549-51.

5 Pinder SE, Rakha EA, Purdie CA, Bartlett JM, Francis A, Stein RC, et al.; Translational Subgroup of the NCRI Breast Clinical Studies Group. Macroscopic handling and reporting of breast cancer specimens pre- and post-neoadjuvant chemotherapy treatment: review of pathological issues and suggested approaches. Histopathology. 2015 Sep;67(3):279-93.

6 Sahoo S, Lester SC. Pathology of breast carcinomas after neoadjuvant chemotherapy: an overview with recommendations on specimen processing and reporting. Arch Pathol Lab Med. 2009 Apr;133(4):633-42.

7 Akashi-Tanaka S, Fukutomi T, Sato N, Iwamoto E, Watanabe T, Katsumata N, et al. The use of contrast-enhanced computed tomography before neoadjuvant chemotherapy to identify patients likely to be treated safely with breast-conserving surgery. Ann Surg. 2004 Feb;239(2):238-43.
8 Amin MB, Edge SB, Greene FL, et al., editors. AJCC Cancer Staging Manual. 8th ed. New York: Springer; 2017.https://doi.org/10.1007/ 978-3-319-40618-3.

9 Aihara T, Toyama T, Takahashi M, Yamamoto Y, Hara F, Akabane H, et al. The Japanese Breast Cancer Society Clinical Practice Guideline for systemic treatment of breast cancer, 2015 edition. Breast Cancer. 2016 May;23(3):329-42.

10 Chollet P, Amat S, Cure H, de Latour M, Le Bouedec G, Mouret-Reynier MA, et al. Prognostic significance of a complete pathological response after induction chemotherapy in operable breast cancer. Br J Cancer. 2002 Apr; 86(7):1041-6.

11 Lakhani SR, Ellis IO, Schnitt SJ, Tan PH, van de Vijver M, editors. WHO classification of tumors of the breast. 2nd ed. Lyon: International Agency for Research of Cancer (IARC); 2012.

12 Naqos N, Naim A, Jouhadi H, Taleb A, Bouchbika Z, Benchakroune N, et al. [Mucinous carcinoma of the breast: Clinical, biological and evolutive profile]. Cancer Radiother. 2016 Dec;20(8):801-4.

13 Ha KY, Deleon P, Deleon W. Invasive mucinous carcinoma of the breast. Proc Bayl Univ Med Cent. 2013 Jul;26(3):295-7.

14 Sharma S, Bansal R, Khare A, Agrawal N. Mucinous carcinoma of breast: cytodiagnosis of a case. J Cytol. 2011 Jan;28(1):42-4.

15 Skotnicki P, Sas-Korczynska B, Strzepek L, Jakubowicz J, Blecharz P, Reinfuss M, et al. Pure and Mixed Mucinous Carcinoma of the Breast: A Comparison of Clinical Outcomes and Treatment Results. Breast J. 2016 Sep; 22(5):529-34.
16 Di Saverio S, Gutierrez J, Avisar E. A retrospective review with long term follow up of 11,400 cases of pure mucinous breast carcinoma. Breast Cancer Res Treat. 2008 Oct; 111(3):541-7.

17 Yang M, Li X, Chun-Hong P, Lin-Ping $\mathrm{H}$. Pure mucinous breast carcinoma: a favorable subtype. Breast Care (Basel). 2013 Mar;8(1): 56-9.

18 Yılmaz TU, Trabzonlu L, Güler SA, Baran MA, Pösteki G, Erçin C, et al. Characteristics of Special Type Breast Tumors in Our Center. Eur J Breast Health. 2018 Jan;14(1):17-22.

19 Lakhani SR, Ellis IO, Schnitt SJ, Tan PH, van de Vijver MJ. WHO Classification of Tumours of the Breast. 4th ed. Lyon: International Agency for Research on Cancer; 2012.

20 Rajan R, Esteva FJ, Symmans WF. Pathologic changes in breast cancer following neoadjuvant chemotherapy: implications for the assessment of response. Clin Breast Cancer. 2004 Aug;5(3):235-8.

21 Rajan R, Poniecka A, Smith TL, Yang Y, Frye D, Pusztai L, et al. Change in tumor cellularity of breast carcinoma after neoadjuvant chemotherapy as a variable in the pathologic assessment of response. Cancer. 2004 Apr; 100(7):1365-73.

22 Nakamura T, Fukutomi T, Tsuda H, AkashiTanaka S, Matsuo K, Shimizu C, et al. Changes in findings of mammography, ultrasonography and contrast-enhanced computed tomography of three histological complete responders with primary breast cancer before and after neoadjuvant chemotherapy: case reports. Jpn J Clin Oncol. 2000 Oct;30(10): 453-7. 